\title{
Design of an air-slot mode-gap nanocavity in a two dimensional photonic crystal slab
}

\author{
REN Cheng ${ }^{1 *}$, KANG Feng ${ }^{2}$, GAN Lin $^{3}$, FENG Shuai ${ }^{4}$, CHENG LiFeng ${ }^{1} \&$ LI ZhiYuan $^{3}$ \\ ${ }^{1}$ School of Opto-Electronic Information Science and Technology, Yantai University, Yantai 264005, China; \\ ${ }^{2}$ Wenjing College, Yantai University, Yantai 264005, China; \\ ${ }^{3}$ Optical Physics Laboratory, Institute of Physics, Chinese Academy of Sciences, Beijing 100190, China; \\ ${ }^{4}$ Academy of Science, Minzu University of China, Beijing 100081, China
}

Received July 16, 2012; accepted September 25, 2012

\begin{abstract}
We present a novel and simple design of an air-slot mode-gap photonic crystal (PC) nanocavity by introducing a linear air slot to the center of a line-defect waveguide in a two dimensional triangular-lattice silicon PC slab. A high quality factor ( $Q$ factor) of $8.42 \times 10^{5}$ and an ultrasmall mode volume of 0.998 cubic wavelength are achieved in an optimized air-slot nanocavity which is a suitable choice for the strong matter-field interaction in free space. The high $Q$ cavities with ultrasmall mode volume are important for applications such as quantum computation and nonlinear optics.
\end{abstract}

photonic crystal slab, air-slot nanocavity, mode gap, quality factor

Citation: $\quad$ Ren C, Kang F, Gan L, et al. Design of an air-slot mode-gap nanocavity in a two dimensional photonic crystal slab. Chin Sci Bull, 2013, 58: 63-67, doi: $10.1007 / \mathrm{s} 11434-012-5610-4$

Recently, two dimensional (2D) silicon photonic crystal (PC) slabs [1-5] have attracted much attention for their in-plane photonic band gap and good compatibility with on chip integration. By introducing point or line defects, nanocavities [6-10], waveguides [11-14], filters [15-17] and various photonic devices can be built in 2D PC slabs. In particular, high quality factor $(Q)$ silicon photonic nanocavities are required for the realization of single photon sources, high resolution filters and strong coupling regime for quantum communication. In view of above applications, it is important to realize the nanocavities having both high $Q$ and small mode volumes $(V)$, which means achieving strong coupling regime between the light and matter in the nanocavities. In order to further enhance the interaction between the light wave and the wave-guiding materials, it is desired to confine light in a narrow active material region. Slot waveguides $[13,14]$ with sub-100 nm-wide air slot offer a good choice, which may be air region or filled with

*Corresponding author (email: cren@ytu.edu.cn) low-refractive-index nonlinear materials. On the basis of above discussion, air-slot PC slab nanocavities [18,19] are designed to achieve strong light confinement region suitable for the study of cavity quantum electrodynamics in free space.

In this work, we present a novel and simple design of mode-gap confined nanocavity by introducing a linear air slot to the center of a line-defect waveguide in a 2D silicon PC slab. Because the normal component of the electric displacement is continuous across the boundary of the air slot wall, the cavity mode is strongly localized inside the slot nanocavity. Correspondingly, we obtain a $Q$ factor of 842000 with a mode volume of 0.998 cubic wavelengths by modifying the tapered region geometry, which is suitable for the applications in the quantum information and quantum optics. Compared to other air slot nanocavities, our design is rather simple and only needs to modify minority parts of air holes around the cavity region for the enhancement of the cavity $Q$ factor. In addition, sub-100 nm-width air slot is more suitable for the fabrication of present micro- 
fabrication precision.

We first investigate the dispersion properties of an airslot PC waveguide to create a larger mode gap region. Then we present the design of our air-slot PC nanocavity confined with mode gap. Strong light confinement is achieved by modifying the tapered region geometry. Finally, we show our theoretical simulation results with different tapered regions.

\section{Nanocavity design and dispersion properties}

We construct an air-slot PC nanocavity confined with mode gap. Figure 1 shows the schematic diagram of our air-slot PC nanocavity. First, we form a W1 PC waveguide by omitting one row of air holes in the $\Gamma-\mathrm{K}$ direction of $2 \mathrm{D}$ triangular lattice photonic crystal built on an air-bridged silicon slab. The thickness of silicon slab $h$ is $0.5465 a$, where $a$ is the lattice constant. Then a $0.163 a$-width air-slot in the $y$ direction is introduced into the center of the line defect of a mode-gap confined PC nanocavity, which is realized by local modulation of the diameters of the two rows of air holes adjacent to the slot waveguide axis. The air holes in the red, green and white boxes, which are shown in Figure 1, are considered as cavity region, tapered regions and mirror regions, respectively. The air holes in the two white boxes are considered as the mirror regions, and their radius is $0.27 a$, the same as the air holes in the background PC. Correspondingly, the two air holes in the red box serve as the cavity region, whose radius is $0.31 a$. The tapered regions in the two green boxes are introduced between the mirror regions and cavity region to localize light more gently, whose radii of three air holes linearly decrease outwards from $0.31 a$ to $0.27 a$. The detailed radii of air holes in the tapered region are $0.30 a, 0.29 a$ and $0.28 a$ outwards respectively. Note that the radii of air holes in the cavity region

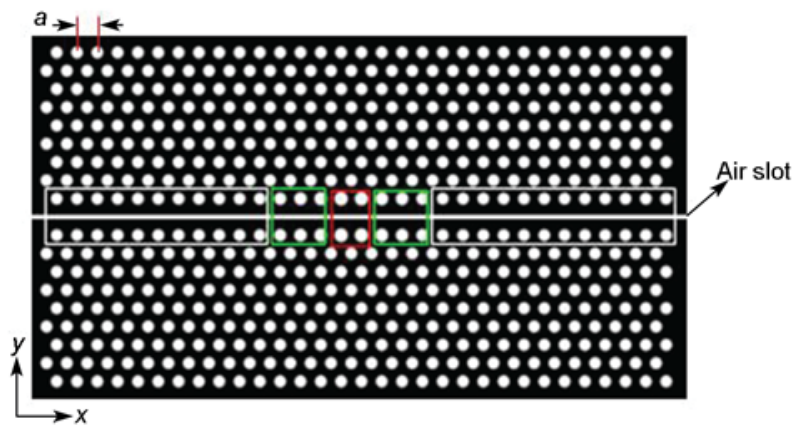

Figure 1 The schematic diagram of an air-slot nanocavity confined with mode gap created in a triangular-lattice photonic crystal slab. The air holes in the red box are considered as cavity region, where the radius is $0.31 a$, where $a$ is the lattice constant. The air holes in the green boxes are linearly decreasing in radius outwards from $0.31 a$ to $0.27 a$, which make up the tapered regions. The radius of the air holes in the two white boxes is $0.27 a$, the same as the air holes in the background. The air slot has a width of $0.163 a$. and mirror regions are chosen as $0.31 a$ and $0.27 a$, respectively, mainly for the reason that the suitable ratio of the airhole radius $r$ to the lattice constant $a$ will result in a larger band gap and working bandwidth, which provides more freedoms and flexibilities in implementing integrated circuits.

The air slot plays a very important role for the realization of strong light confinement in free space. To have a basic idea about the air-slot PC waveguide, we first tailor the dispersion properties of the designed air-slot PC waveguide. We employ the well-established three-dimensional planewave expansion method [20] to calculate the band structures of the background PC slab and air-slot PC guided modes. In the simulations, 32 pixels per lattice constant $a$ together with periodic boundary condition are used and good accuracy is ensured. The PC slab exhibits a $2 \mathrm{D}$ band gap of TElike surface modes ranging from $0.2623 a / \lambda$ to $0.3165 a / \lambda$. The calculated band diagrams with TE-like polarization for the $0.163 a$-width air-slot PC waveguide are shown in Figure 2(a). In the simulation, a supercell is used, whose schematic configuration is illustrated in Figure 2(b). For the sake of clarity, the leaky region above light cone and the pass band regions above and below the band gap are all filled with light gray color. There are altogether two guided slot waveguide modes within the whole band gap, which are designated as modes 1 and 2 by the black lines with triangular and circle points, respectively. Modes 1 and 2 can be classified to the fundamental even mode and higher-order odd mode in the band gap, respectively, according to the symmetry of the $E_{y}$ field component with respect to the mirror reflection plane passing through the waveguide central axis. Note that the fundamental even mode 1 shows a

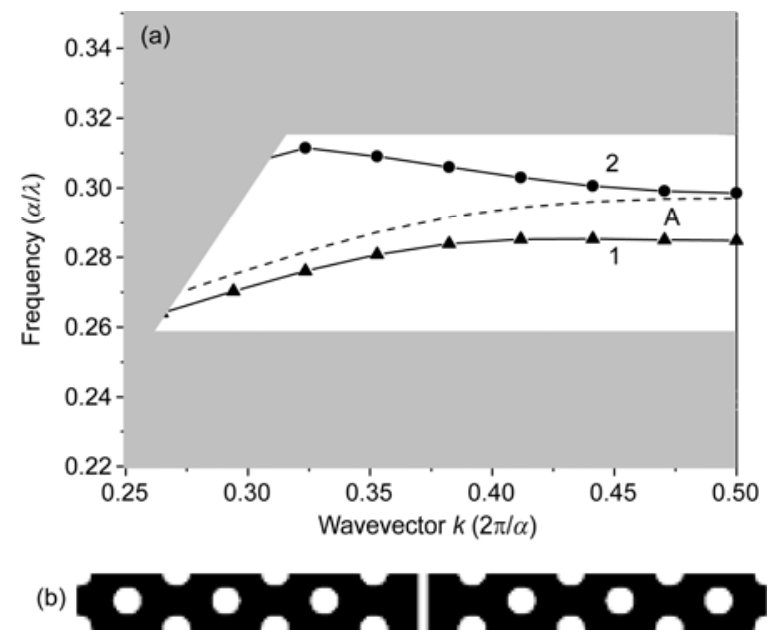

Figure 2 (a) The band diagrams for the $0.163 a$-width air-slot PC waveguide simulated by $3 \mathrm{D}$ plane-wave expansion method. The black triangular and circle dot lines correspond to the guided modes (modes 1 and 2) for the background air-slot waveguide with the radius of air holes being $0.27 a$, while the dash line (mode A) corresponds to the guided mode of the cavityregion air-slot waveguide with the radius of air holes as $0.31 a$. (b) The supercell model of the $0.163 a$-width air-slot PC waveguide with the radius of air holes being $0.27 a$. 
positive slope in the first Brillouin zone. Accordingly, a mode-gap region is formed between modes 1 and 2, which we can use to construct a high $Q$ nanocavity when selecting suitable working frequency of the nanocavity. The geometric parameters of the $\mathrm{PC}$ slab structure we designed are as follows: the air hole radius $r$ is $0.27 a$, the width of air-slot in the $\mathrm{y}$ direction is $0.163 \mathrm{a}$, the thickness of silicon slab $h$ is $0.5465 a$, and the refractive index of the background silicon is 3.4 .

As described in [11], the even PBG modes can be tuned when changing the diameter of the first row of air holes in the $\mathrm{W} 1 \mathrm{PC}$ waveguide. So when we change the radius of the two rows of air holes adjacent to the slot waveguide axis to $0.31 a$, the slot waveguide modes can be tuned to the higher frequency. Consequently, the air-slot even mode 1 (now designated as mode A) still lies within the photonic band gap as shown by the red line in Figure 2(a), while the odd mode 2 moves to the higher frequency and mixes with the air band edge, which is not shown in Figure 2(a) because it does not concern us. By comparing the band diagram of the cavity and barrier region of the air-slot waveguide, as displayed in Figure 2(a), we find that part of the frequency of the even mode $\mathrm{A}$ in the cavity region is located within in the mode gap region of the confinement region. Therefore, this can be a suitable choice for the working frequency region of slot nanocavity. Besides, the nanocavity has a rather large working bandwidth which supports a single mode from $0.2848 a / \lambda$ to $0.2985 a / \lambda$ when the air slot width is selected as $0.163 a$.

Similarly, we calculate the band structures of $0.140 a$ width air-slot PC waveguides with radius $0.27 a$ of air holes in the background PC and $0.31 a$ of air holes adjacent to the slot waveguide axis, respectively, and then merge them into one as shown in Figure 3(a). It is evident that the frequency of mode $\mathrm{A}$ in the mode gap region range from $0.2802 \mathrm{a} / \lambda$ to $0.2906 a / \lambda$, which has a smaller working bandwidth than the corresponding $0.163 a$-width air-slot PC waveguides. Accordingly, it will provide less freedoms and flexibilities in implementing integrated circuits. In the same way, when changing the slot width to $0.186 a$ while keeping other parameters the same as before, the calculated band structure is shown in Figure 3(b). The results show that mode A exceeds the mode gap region and mixes with mode 2 , which may cause the annoying multimode region. In short, the $0.163 a$-width air slot PC is an optimal selection.

\section{Optical properties of the air-slot nanocavity}

The normalized resonant frequency of the air-slot nanocavity is $0.290082 a / \lambda$ calculated by 3D-FDTD simulation [21], which corresponds to the slot waveguide even mode $\mathrm{A}$ in the mode gap region as shown in Figure 2(a). In the simulations, 22 pixels per lattice constant $a$ together with Berenger's perfectly matched layer (PML) boundary condition
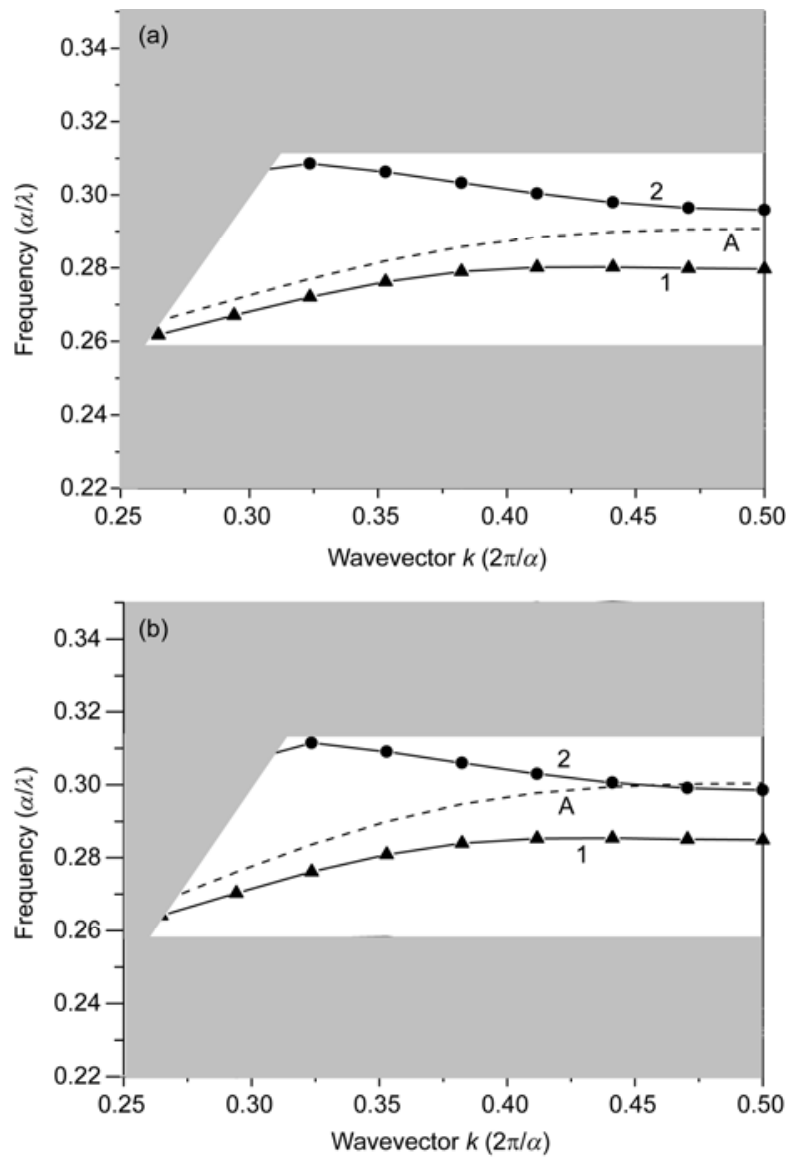

Figure 3 The combined band diagrams of air-slot PC waveguides with radius $0.27 a$ of air holes in the background PC and $0.31 a$ of air holes adjacent to the slot waveguide axis, respectively. (a) $0.140 a$-width air-slot PC waveguide; (b) $0.186 a$-width air-slot PC waveguide. Modes 1 and 2 correspond to the guided modes for the background air-slot waveguide with the radius of air holes being $0.27 a$, while mode A corresponds to the guided mode of the cavity-region air-slot waveguide with the radius of air holes as $0.31 a$.

are used, which is enough for ensuring the good accuracy. Using Pade approximation combined with 3D-FDTD simulation, we obtain $Q=8.42 \times 10^{5}, V=0.998$ in units of a cubic wavelength. The calculated line shape of the air-slot cavity mode is shown in Figure 4(a). The black line is the result of the Pade approximation, and the red line is the Lorentz fit of the black curve. A point source of continuous wave resonating at $0.290082 a / \lambda$ is placed into the cavity and located near the center of the cavity. Figure 4(b) shows the distribution of the electric field $E_{y}$ for the cavity mode in the central plane of the silicon slab, which has even symmetry with respect to the mirror refection plane passing through the slot waveguide central axis. It is evident that electric field of cavity mode is strongly localized in the free space region, which is suitable for the study of matter-field interaction.

In order to further improve the performance of the airslot nanocavity, we investigate the effects of different lengths and air hole sizes of the tapered regions on the quality factor $Q$ and mode volume $V$. In the above simulation, there 

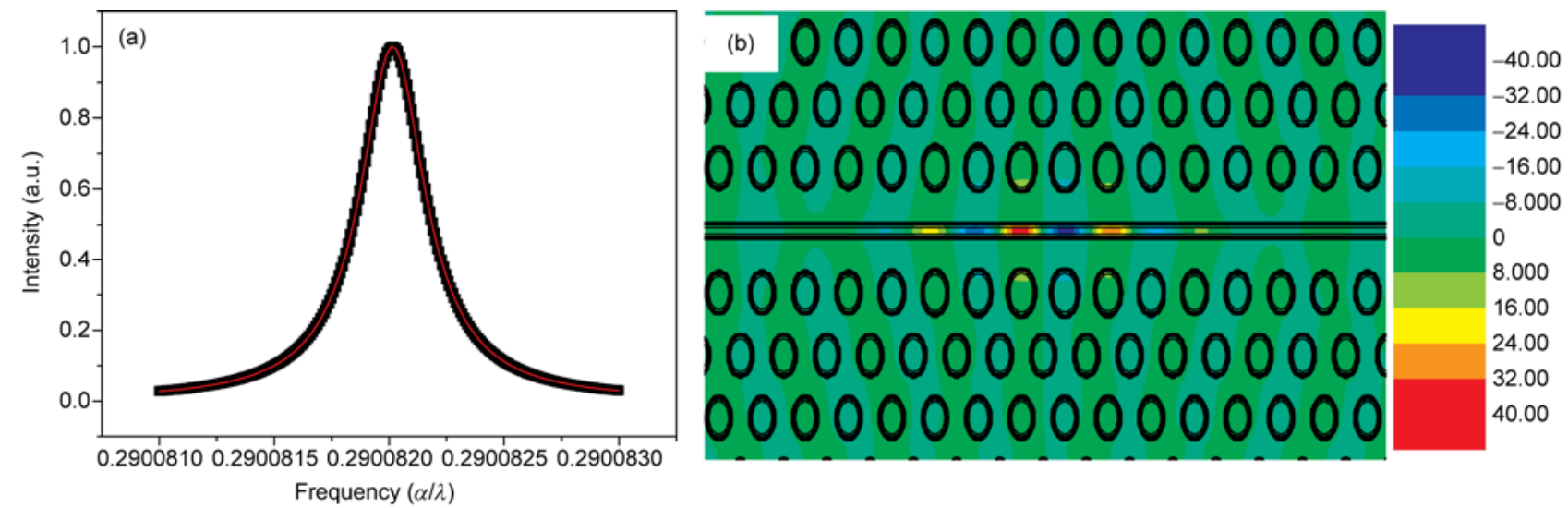

Figure 4 (a) Calculated resonance spectrum for the cavity mode of the air-slot nanocavity shown in Figure 1. The black line is the results of the Pade approximation, and the red line is the Lorentz fit of the black line. (b) $E_{y}$ field distribution of the corresponding cavity mode in the central plane of the silicon slab.

Table $1 Q$ and $V$ of air-slot cavities with different numbers of air holes in the tapered regions

\begin{tabular}{cccc}
\hline Number of air holes in each tapered region & $Q$ factor & Mode volume $V$ (cubic wavelength) & $Q / V$ \\
\hline 0 & $2.88 \times 10^{4}$ & 1.347 & $2.138 \times 10^{4}$ \\
1 & $1.14 \times 10^{5}$ & 1.116 & $1.022 \times 10^{5}$ \\
2 & $4.24 \times 10^{5}$ & 1.075 & $3.944 \times 10^{5}$ \\
3 & $8.42 \times 10^{5}$ & 0.998 & $8.437 \times 10^{5}$ \\
4 & $6.41 \times 10^{5}$ & 1.089 & $5.886 \times 10^{5}$ \\
\hline
\end{tabular}

are 3 air holes in each tapered region. Now we simulate different tapered regions with $0,1,2$, and 4 air holes on each side. Note that the radii of air holes in the tapered region linearly decrease outwards from $0.31 a$ to $0.27 a$. The radii of the air holes in the mirror regions and cavity region are the same as above, $0.27 a$ and $0.31 a$, respectively. The detailed results are shown in Table 1 . We can see that the tapered region with 3 air holes offers the largest $Q / V$, while the tapered region without any air hole (namely, with no tapered region) offers the smallest $Q / V$. Actually, when the electric field of the cavity mode is close to a Gaussian distribution, a very high $Q$ factor can be achieved and so the above difference in $Q / V$ is easy to understand.

\section{Conclusion}

We have designed a novel and simple air-slot mode-gap nanocavity in 2D silicon PC slabs. The calculated quality factor is as high as $8.42 \times 10^{5}$ and the mode volume is as small as 0.998 cubic wavelengths by modifying the geometry of the nanocavity. These results clearly show that the designed slot nanocavity with ultrasmall mode volume is a suitable choice for the applications such as quantum computation and nonlinear optics.

This work was supported by the National Natural Science Foundation of China (11004169 and 10904176) and the Doctoral Foundation of Shandong Province (BS2009CLO28).
1 Yablonovitch E. Inhibited spontaneous emission in solid-state physics and electronics. Phys Rev Lett, 1987, 58: 2059-2062

2 John S. Strong localization of photons in certain disordered dielectric superlattices. Phys Rev Lett, 1987, 58: 2486-2489

3 Noda S, Chutinan A, Imada M. Trapping and emission of photons by a single defect in a photonic bandgap structure. Nature, 2000, 407: 608-610

4 Tokushima M, Yamada H. Light propagation in a photonic-crystalslab line-defect waveguide. IEEE J Quant Electr, 2002, 38: 753-759

5 Liu Y Z, Liu R J, Zhou C Z, et al. $\Gamma$-M waveguides in two-dimensionaltriangular-lattice photonic crystal slabs. Opt Express, 2008, 16: 21483-21491

6 Kuramochi E, Notomi M, Mitsugi $\mathrm{S}$, et al. Ultrahigh- $Q$ photonic crystal nanocavities realized by the local width modulation of a line defect. Appl Phys Lett, 2006, 88: 041112

7 Zhou C Z, Xiong Z G, Li Z Y. High- $Q$ microcavity in two-dimensional diamond photonic crystal thin films realized via a mode gap. Chin Phys Lett, 2009, 26: 094201

8 Song B S, Noda S, Asano T, et al. Ultra-high- $Q$ photonic doubleheterostructure nanocavity. Nat Mater, 2005, 4: 207-210

9 Zhang Z Y, Qiu M. Small-volume waveguide-section high $Q$ microcavities in 2D photonic crystal slabs. Opt Express, 2004, 12: 39883995

10 Robinson J T, Manolatou C, Chen L, et al. Ultrasmall mode volumes in dielectric optical microcavities. Phys Rev Lett, 2005, 95: 143901

11 Lars H F, Andrei V L, Jacob F P, et al. Photonic crystal waveguides with semi-slow light and tailored dispersion properties. Opt Express, 2006, 14: 9444-9450

12 Liu Y Z, Liu R J, Feng S, et al. Multichannel filters via $\Gamma-\mathrm{M}$ and $\Gamma-\mathrm{K}$ waveguide coupling in two-dimensional triangular-lattice photonic crystal slabs. Appl Phys Lett, 2008, 93: 241107

13 Wu J, Peng C, Li Y P, et al. Wideband slow light in chirped slot photonic-crystal coupled waveguides. Chin Phys Lett, 2009, 26: 014209

14 Falco A D, Faolain L O, Krauss T F. Photonic crystal slotted slab waveguides. Photon Nanostruct, 2008, 6: 38-41 
15 Shinya A, Mitsugi S, Kuramochi E, et al. Ultrasmall multi-channel resonant-tunneling filter using mode gap of width-tuned photoniccrystal waveguide. Opt Express, 2005, 13: 4202-4209

16 Ren C, Tian J, Feng S, et al. High resolution three-port filter in two dimensional photonic crystal slabs. Opt Express, 2006, 14: 10014-10020

17 Takano H, Song B S, Asano T, et al. Highly efficient multi-channel drop filter in a two-dimensional hetero photonic crystal. Appl Phys Lett, 2005, 86: 241101

18 Yamamoto $\mathrm{T}$, Notomi M, Taniyama $\mathrm{H}$, et al. Design of a high- $Q$ air-slot cavity based on a width-modulated line-defect in a photonic crystal slab. Opt Express, 2008, 16: 13809-13817

19 Gao J, Mcmilan J F, Wu M C, et al. Demonstration of an air-slot mode-gap confined photonic crystal slab nanocavity with ultrasmall mode volumes. Appl Phys Lett, 2010, 96: 051123

20 Johnson S G, Joannopoulos J D. Block-iterative frequency-domain methods for Maxwell's equations in a planewave basis. Opt Express, 2001, 8: 173-190

21 Taflove A, Hagness S C. Computational Electrodynamics: The FiniteDifference Time-Domain Method. 2nd ed. Boston: Artech House Publishers, 2000

Open Access This article is distributed under the terms of the Creative Commons Attribution License which permits any use, distribution, and reproduction in any medium, provided the original author(s) and source are credited. 